\title{
Reconfiguration de l'organisation : suivre à la trace les figures textualisées - le cas de la figure du patient
}

Reconf iguring the Organization: Representations of the Patient in Quality

Healthcare Discourse

\section{Alexia Jolivet et Consuelo Vásquez}

\section{OpenEdition}

Journals

Édition électronique

URL : http://journals.openedition.org/edc/2563

DOI : 10.4000/edc. 2563

ISSN : 2101-0366

Éditeur

Université Lille-3

Édition imprimée

Date de publication : 1 juin 2011

Pagination : $129-146$

ISBN : 978-2-917562-05-5

ISSN : 1270-6841

\section{Référence électronique}

Alexia Jolivet et Consuelo Vásquez, «Reconfiguration de l'organisation : suivre à la trace les figures textualisées - le cas de la figure du patient », Études de communication [En ligne], 36 | 2011, mis en ligne le 08 juillet 2011, consulté le 19 avril 2019. URL : http://journals.openedition.org/edc/2563; DOI : $10.4000 /$ edc. 2563

Ce document a été généré automatiquement le 19 avril 2019.

(c) Tous droits réservés 


\title{
Reconfiguration de l'organisation : suivre à la trace les figures textualisées - le cas de la figure du patient $^{1}$
}

\author{
Reconf iguring the Organization: Representations of the Patient in Quality \\ Healthcare Discourse
}

Alexia Jolivet et Consuelo Vásquez

\section{Introduction ${ }^{2}$}

Dans un ouvrage récent, Cooren (2010a) développe la notion de ventriloquie, notion selon laquelle la communication en situation de dialogue impliquerait de faire parler des figures et aussi de parler aux noms de celles-ci. La mise en scène de figures multiples textuelles, humaines, architecturales, artéfactuelles - dans des textes (écrits ou oraux) rend compte du mode d'existence pluriel de l'organisation, qui doit alors être conçu comme étant le résultat d'une configuration d'agentivité hybrides (Cooren, 2006 ; Brassac, Fixmer, Mondada et Vinck, 2008; Brassac et Le Ber, 2005). Dès lors il importe de comprendre, comme nous suggère Cooren (2010b), comment les textes écrivent l'organisation - ce que nous proposons de développer dans cet article dans un (con-)texte particulier, celui des démarches qualités dans le secteur hospitalier en France.

2 Les démarches qualité ont suscité de nombreux travaux quant à leurs conséquences organisationnelles. Dans le domaine hospitalier, la question de la qualité est fortement liée à celle de la mise sous contrainte des ressources du système hospitalier par les financeurs publics (Minvielle, 2003, Pierru, 2009), et ce depuis 1975. Dans ce contexte, la grande interrogation qui se pose est de savoir si la réduction ou la modération de l'évolution des coûts ne se traduisent pas par des soins de moins bonne qualité (Mayère et 
al., 2010, Castel, 2002, Sainsaulieu, 2007, Paccioni et al., 2010). De nombreuses réformes ont essayé d'y répondre : les ordonnances Juppé de 1996, la création de l'Agence Nationale d'Accréditation et d'Évaluation en Santé. Mais aussi des lois qui mettent le patient au cœur des démarches: la loi de 2002 sur le droit des malades, qui met en place des dispositifs prenant en compte le point de vue des usagers tels que les enquêtes de satisfaction, la participation aux instances de décision interne, la création de la C ommission des Relations avec les Usagers et de la Qualité de la prise en Charge.

Ce contexte nous invite à prendre en compte la dimension politico-étatique des démarches qualité. Ogien (2009) y souligne l'ambivalence de la notion de «qualité » qui renvoie à la fois à une évaluation exprimant une mesure (une prestation de bonne ou de mauvaise qualité) et à une propriété qui échappe à toute mesure (on oppose qualité à quantité). Deux usages dominants sont donc en tension dans ces processus : l'un est moral, l'autre économique et managérial, ce dernier inscrivant la qualité comme un dispositif technique visant à optimiser l'efficacité de la médecine. Ogien conclut en constatant l'accroissement, dans une optique de régulation étatique des établissements, de l'emprise de la quantification sur l'univers des pratiques hospitalières.

Notre article cherche à contribuer à cette réflexion autour des démarches qualité dans le secteur hospitalier et plus particulièrement à cerner la relation entre l'organisation de soins de santé et le patient. Or, notre positionnement diffère des travaux cités précédemment dans la mesure où notre propos s'attache à cerner les configurations de l'organisation suscitées par l'emploi de la figure du patient dans des textes normatifs, plus précisément ceux de la Haute Autorité de Santé (HAS) de France ${ }^{3}$. Nous ne nous intéressons pas à la mise en pratique de ces démarches dans les organisations mais aux postulats et (pré)configurations supposés inscrites dans ces textes. Néanmoins, notre étude ne peut ignorer les problématiques évoquées dans les travaux cités. Nous nous penchons alors sur les textes comme étant à la fois des inscriptions et des agents de l'organisation participant de la complexité des démarches qualité. Les textes sont ici définis comme médiateurs, leur «faire-faire » (Bouzon, 2009 ; Cooren, 2004 ; Morillon, 2009 ; Pontille, 2009) est associé aux multiples figures - ce que nous nommons des figures textualisées - qui donnent du poids (font autorité) au discours des individus et des organisations.

5 Nous proposons dans cet article de retracer les figures du patient dans des textes de qualité et, par ce biais, y questionner sa place : où est le patient? Est-il placé en tant qu'usager, client, consommateur, participant, expert ? Les démarches qualité poussent les organisations de soins de santé à s'ouvrir vers cet acteur: à se déplier - se laisser questionner par (et pour) le patient - et à s'y (re)plier - se renfermer, y envelopper le patient. Or la manière dont ses figures sont mises en acte (ou plutôt " mises en écrit ", Fraenkel, 2007) dans les textes qualité laisse entrevoir que bien que le patient soit au cœur de ces démarches, c'est l'organisation et sa reconfiguration qui sont au cœur des problématiques. En effet, par l'appel à la figure du patient, c'est moins le patient que l'organisation qui est questionné. Nous cherchons, dès lors, à saisir comment derrière l'acception " patient » se cachent de multiples figures, elles-mêmes à autorité différente selon les configurations qu'elles mettent en jeu. Cette multiplicité pose ainsi un enjeu pour ces organisations de santé : à l'image de la figure du patient, doit-elle devenir multiple?

6 Notre propos est de montrer le lien intrinsèque entre figure du patient et configuration de l'organisation en tant que co-constructions. Nous faisons l'hypothèse que l'étude des 
figures textualisées du patient est une clé pour comprendre les mouvements de pli et de dépli au travers desquels le patient et l'organisation sont reconfigurés; les figures du patient poussent ainsi l'organisation à s'« espacer » différemment (Vasquez, 2009).

\section{Sur les figures et les plis... pour espacer l'organisation}

7 La notion de figure renvoie étymologiquement à quelque chose qui est fabriquée, qui est modelée. Il faut donc la produire, la façonner pour qu'elle existe. Cooren (2010a) souligne ce caractère de production et re-production de la figure. Dans ce sens, pour lui, la figure implique une re-présentation. Non seulement la figure doit être mise en présence pour être reconnue comme telle (une mise en présence qui peut prendre diverses formes), mais c'est aussi par la mise en présence que la figure opère. La figure devient alors une forme d'agentivité (un principe, une valeur, une passion, une règle, un objet, etc.) présentée - ou plutôt re-présentée - dans le discours. Pour Cooren les figures sont d'excellentes ressources discursives: elles permettent de "donner du poids» au discours en y incorporant une diversité d'agents. C'est par l'association des figures - ce que nous nommons des (re)configurations - qu'ainsi, par exemple, le chef de services, la norme de santé, le principe de bientraitance, l'association des usagers sont articulés autour de/avec le patient. Ces figures agissent dans le discours : elles font autorité - elles sont auteur et elles autorisent - et la concèdent (ou pas) au patient selon la manière dont elles sont configurées.

Ce sont ces (re-)configurations - ces agencements de figures que nous étudions dans les textes qualité - qui poussent l'organisation à se (re-)plier et à se déplier : le dépli n'étant pas le contraire du pli, mais sa continuité. Il intéresse alors de comprendre, comme l'explique Deleuze (Deleuze, 1988), ce mouvement du pli au dépli ; c'est-à-dire de l'enveloppement à l'inclusion. Plier, déplier, replier l'organisation signifie, en reprenant les termes de Vasquez (Vasquez, 2010) espacer l'organisation, action qui est rendue possible par les figures. En effet, c'est par l'association de principes, de valeurs, de personnes, d'objets incarnés dans les figures que nous reconfigurons l'organisation : nous la plions, la déplions, la replions selon d'éventuelles inclusions/exclusions. Si nous acceptons que le pli soit l'expression minimale de l'organisation, ce qui permet de plier, de déplier et de replier l'organisation ce sont les agencements de figures: ces reconfigurations.

9 Pour étudier ces mouvements de reconfigurations, nous nous centrons sur les textes que nous définissons ici en tant que (1) inscriptions d'un processus de négociation et de collaboration (Joerges et Czarniawska, 1998) entre les différentes parties prenantes associées aux processus d'accréditation des établissements de santé en France mené par la HAS et (2) en tant qu'agents (Cooren, 2004) qui participent à la mise en écrit (Fraenkel, 2007) des figures du patient et, par là-même, de l'organisation de soins de santé. En retraçant les différentes figures du patient dans ces textes qualité nous sommes amenés à y questionner sa place : est-il inclus ou exclu, enveloppé ou développé par l'organisation? Notre analyse suggère, comme nous le verrons par la suite, que, bien que dans certains cas le patient soit plié par l'organisation, dans d'autres c'est lui qui la plie et la déplie, ce faisant la reconfigure. Nous devons noter que l'analyse qui suit se veut illustrative d'une démarche conceptuelle pour suivre à la trace les figures textualisées et leurs agencements. Il ne s'agit pas d'une étude empirique sur les textes qualité en milieu hospitalier, mais plutôt d'une mise en application d'un développement conceptuel. 


\section{(Re-)configurations du patient dans les textes qualités : comment l'organisation se plie et se déplie}

10 Les textes que nous avons choisis pour l'analyse des figures du patient sont de caractère normatif (ou en découlent). L'énonciateur de ces textes - la HAS - n'est pas anodin ; il fait autorité en tant qu'instance élaboratrice de prescriptions normatives afin de réguler le système de santé. Ces textes s'inscrivent ainsi dans une démarche normative, une approche qui nous semblait pertinente à étudier, non pas dans l'optique théorique d'une analyse politico-symbolique des démarches qualité, mais dans une volonté méthodologique de s'attacher aux figures dans leur agentivité - la normativité étant une des configurations des plus révélatrices et des plus saisissables. Nous avons ensuite sélectionné les textes dans lesquels transparaissaient, de manière prégnante, différentes figures du patient. Engagés dans une première étape exploratoire, nous en avons dégagés trois et nous nous sommes concentrés sur les textes les mettant plus particulièrement en scène.

11 Le premier texte correspond au manuel de certification des établissements de santé version 2010; il présente le dispositif actuel de certification en France à travers deux grands axes: le management de l'établissement et la prise en charge du patient. C'est surtout le deuxième axe que nous avons analysé sous l'angle des droits du patient. Le deuxième texte intitulé "Chemin clinique : une méthode d'amélioration de la qualité " présente la description d'une pathologie donnée en suivant le parcours type du patient de manière à structurer les différents éléments du processus de sa prise en charge. Le troisième texte aborde le thème des associations de patients et d'usagers dans un guide intitulé "Cadre de coopération avec les associations de patients et d'usagers » qui systématise le rôle de ces associations, leur niveau d'implication et les étapes de consultation et coopération. Ces trois textes nous donnent un premier portrait des figures du patient mobilisées par la HAS. Nous avons cependant considéré qu'il serait intéressant de voir comment ces figures sont traduites dans d'autres (con-)textes et vis-à-vis d'autres interlocuteurs/lecteurs. Pour ce faire, nous avons choisi un article produit par une cellule qualité d'un centre de soin de santé en Midi Pyrénées ainsi qu'un communiqué de presse produit par la HAS concernant la consultation en ligne des citoyens sur le processus d'accréditation.

Dans les textes que nous avons étudiés, nous nous attachons à cerner les différentes figures à l'œuvre et convoquées pour en comprendre les incidences sur le déploiement dépliement - (et repliement) de l'organisation. Le tableau ci-dessous associe les textes étudiés aux figures textualisées du patient et aux re-configurations de l'organisation. 
TABLEAU 1 : FIgURES TEXTUALISÉES DU PATIENT ET RECONFIgURATIONS DE LA HAS

\begin{tabular}{|c|c|c|}
\hline Textes: & $\begin{array}{c}\text { Figures textualisées } \\
\text { du patient }\end{array}$ & $\begin{array}{l}\text { Re-configurations } \\
\text { de la } \text { HAS }_{\#}\end{array}$ \\
\hline $\begin{array}{l}\text { Manuel de certification des } \\
\text { établissements de santé } \\
(2010)^{\#}\end{array}$ & Le patient-légal" & $\begin{array}{l}\text { L'organisation-morale| } \\
\text { L'organisation-éthique }\end{array}$ \\
\hline $\begin{array}{l}\text { Chemin clinique : une } \\
\text { méthode d'amélioration de } \\
\text { la qualité }\end{array}$ & $\begin{array}{l}\text { Le patient-client } \\
\text { Le patient-parcours } \\
\text { Le patient-argument }\end{array}$ & $\begin{array}{l}\text { L'organisation-fournisseur } \\
\text { L'organisation-instrument }\end{array}$ \\
\hline $\begin{array}{l}\text { Cadre de coopération avec } \\
\text { les associations de patients } \\
\text { et d'usagers" }\end{array}$ & 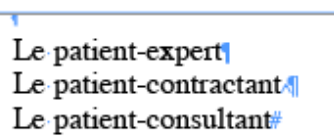 & $\begin{array}{l}\text { L'organisation - contractant } \| \\
\text { L'organisation - cadre \# }\end{array}$ \\
\hline $\begin{array}{l}\text { Communiqué de presse de } \\
\text { la HAS\# }\end{array}$ & $\begin{array}{l}\text { Le patient-contributeur } \\
\text { Le patient-citoyen }\end{array}$ & $\begin{array}{l}\text { L'organisation qui consulte| } \\
\text { L'organisation qui décide }\end{array}$ \\
\hline Article de cellule qualité & $\begin{array}{l}\text { Le patient-client } \\
\text { Le patient-livrable }\end{array}$ & $\begin{array}{l}\text { L'organisation-fournisseur| } \\
\text { L'organisation-instrument }\end{array}$ \\
\hline
\end{tabular}

\section{Le patient-expert : déplier l'organisation... sous certaines conditions}

13 Dans le cadre de sa fonction, la HAS diffuse des guides d'aide aux différents acteurs du monde de la santé. Le guide "Cadre de coopération avec les associations de patients et d'usagers $\|^{5}$ définit les conditions de coopération de la HAS avec les associations de patients. Dans le cadre de ses missions, la HAS souligne qu'elle se doit de mener des « expertises techniques aux conséquences importantes pour l'ensemble des acteurs de la santé » (p. 1). A ce titre, les patients sont considérés comme des acteurs légitimes d'être entendus. Néanmoins, cette consultation des patients est médiatisée: ce sont les associations de patients et d'usagers qui sont convoquées. Le guide se présente comme un ensemble de conseils à valeur normative d'intégration de ces acteurs dans le cadre des activités de la HAS. La présentation de ces conseils est également une manière pour la HAS de valoriser ses principes d'action: la reconnaissance et la formalisation de l'expertise des patients et usagers, l'harmonisation des règles de sélection des associations, la transparence des règles de sélection et de fonctionnement de cette coopération. Deux temps se dégagent de ces principes. Le premier est la reconnaissance $\mathrm{du}$ patient en tant qu'expert. En cela, le patient est également détenteur de savoirs susceptibles d'enrichir le travail de la HAS. Cette expertise change le statut du patient qui passe de bénéficiaire de soin à témoin et spécialiste. Le deuxième temps souligne la formalisation de la relation avec la HAS. Si le patient ou ses représentants sont légitimes en tant qu'interlocuteurs, cette légitimité est soumise à caution selon des principes de sélection et de règles de fonctionnement. Ainsi, il est marquant de voir dans ce guide que lorsque la figure du patient en tant qu'expert apparaît, elle n'est nullement définie comme la valorisation d'individus détenteurs de savoirs spécifiques, mais selon les droits et les responsabilités que cela implique. La notion de « cadre » n'en est que plus accentuée.

La figure d'expert mobilisée ici pousse la HAS, et par conséquent l'ensemble des établissements de santé, à s'ouvrir à de nouveaux interlocuteurs, voire à se déplier afin de rendre accessible à ces nouveaux acteurs des facettes de l'organisation restées repliées. 
Ces nouveaux acteurs, qui paradoxalement n'en sont pas par rapport au système de santé qui les a soignés, peuvent dès lors agir en amont des organisations en participant aux travaux de la HAS selon plusieurs modalités : être à titre de membre des commissions, des groupes de travail ou encore des groupes de lecture des documents. Un nouvel espace de parole s'ouvre à eux; mais un espace bien gardé. En effet, s'il y a reconnaissance de l'expertise du patient, une volonté de formaliser cette expertise est patente. Outre l'accès filtré des associations (la « HAS choisit elle-même les associations de patients et d'usagers auxquelles elle propose de participer à des travaux» p. 3), la relation patient-HAS est contractualisée : être expert implique certaines obligations dont le respect détermine la viabilité du contrat entre les deux actants. Ce contrat repose sur la rédaction de "Déclarations Personnelles d'Intérêts", sur le respect de la confidentialité des documents intermédiaires et sur un principe de rémunération. Le patient devient consultant, ce qui n'est pas sans soulever des questions de (im)partialité. La figure présentée ici est donc ambigüe: un patient à la fois reconnu et considéré comme potentiel auteur (il peut participer à la rédaction de documents, et il fait autorité en tant qu'expert) et à la fois cadré dans son apport à l'organisation. Si l'organisation se déplie pour le nouvel acteur, ce dernier se plie à l'organisation pour pouvoir en faire partie.

Toute figure est le résultat d'une configuration - un ensemble de valeurs et de principes qui s'imbriquent pour lui donner corps. Ici, la figure du patient-expert est associée à une reconnaissance d'une compétence, l'expertise, qui est définie par un ensemble de droits et de devoirs. Dans cet agencement, cette figure est donc fortement en lien avec celle de la HAS, elle est soumise à cette instance. La figure du patient-expert se définit par le cadre établi par la HAS.

Derrière cette ouverture de l'organisation, cette figure du patient comme expert, pourrait-on y voir les prémices d'une figure du patient en tant que citoyen? Le communiqué de presse de la $\mathrm{HAS}^{6}$ annonçant la mise en consultation publique de documents fait référence à ce nouveau niveau d'action évoqué ci-dessus: une participation rédactionnelle du patient comme auteur/autorité. Cette consultation ouverture publique est, comme son nom l'indique, une demande d'avis mais n'a pas en soi de valeur décisionnelle. Les associations sont présentées comme des "contributeurs", une qualification qui légitime leur apport mais qui les maintient au statut d'aidant. Quelle figure du patient se cache-t-il dès lors? Celle du patient-expert? Celle du patientcitoyen? Ou celle d'un patient-client (qui vient tester le manuel)?

\section{De la clientélisation du patient ou le patient devenu parcours : plier l'organisation}

17 Nous avons pu relever l'établissement d'une première forme de relation entre le patient et la HAS : la relation contractuelle entre l'expert et l'organisation. Nous allons voir que ce type de relation peut prendre une nouvelle forme à un autre niveau : c'est la relation client-fournisseur.

Dans "Chemin clinique : une méthode d'amélioration de la qualité »", la HAS prodigue une méthode qui vise à "planifier, rationaliser et standardiser la prise en charge pluridisciplinaire de patients présentant un problème de santé comparable» (p. 2 du guide raccourci). Centré autour du patient, le chemin clinique a pour but d'optimiser les ressources, d'améliorer la coopération des différents acteurs au sein de l'établissement et l'efficacité de la prise en charge. Ce chemin clinique, dans sa forme la plus aboutie, se 
présente sous la forme d'un dossier papier ou informatisé où chaque étape de la prise en charge et les actes à réaliser qui en découlent sont décrits et tracés. Il s'agit donc de planifier le parcours du patient durant sa prise en charge, en identifiant ses étapes clés. Dans cette focale, la figure du patient se présente donc comme une trajectoire scriptée (Vasquez, 2009, à venir) - en tant que script principal-autour desquelles se reconfigurent les trajectoires des membres du personnel. Ces derniers se définissent donc moins par leur métier que par leur contribution au parcours du patient. Le Chemin clinique contribue à l'ordonnancement du personnel par rapport au patient. Or, si le patient, ou plutôt son parcours, est l'élément central de cette méthode, il en est paradoxalement absent. En effet, l'élaboration du chemin clinique repose sur les actions de groupes de travail composés par l'ensemble des professions y intervenant. Le médecin, l'infirmier, l'aide soignant, le psychologue, tous sont présents... sauf le patient.

Si donc ce dernier est absent en tant que personne ou acteur, quelle figure nous en estelle donnée ? La figure du patient est tout d'abord citée en tant qu'argument : le chemin clinique augmente les bénéfices du patient (pp. 5, 27). C'est donc le renforcement des valeurs de service et d'aide que la figure du patient comme bénéficiaire mobilise. Mais finalement, ce n'est que dans la partie introductive que cette figure est présente ; après, plus de traces... ou presque : on le retrouve dans des séries d'actes de soin qui réfèrent à son corps, à son anxiété, à la diffusion d'informations à son égard. La construction du parcours du patient renvoie à une trajectoire type, voire à un patient type. Il est demandé à l'organisation de textualiser le patient, tout comme le fait ce guide : ce qui rend la figure du patient générique, abstraite. Ce patient type devient le patient de référence ; celui qui présente un écart - le vrai, la personne - en est exclu. L'organisation se plie et se déplie selon cette trajectoire scriptée, mais, de ce fait, aussi, inclut et exclut selon les critères établis par cette trajectoire.

On en arrive donc à une situation où le texte agit en tant que document de traçabilité, d'articulation, d'organisation et de contrôle. La figure du patient est ainsi configurée autrement: ce n'est plus le patient en tant qu'acteur qui est évoqué, mais une représentation d'un patient générique. La figure du patient analysée ici est différente de celle du patient-expert, elle implique d'autres configurations, qui lui donnent une autorité différente.

Il est à noter que derrière la figure du patient comme parcours se détache la figure du patient comme axe stratégique de la démarche qualité. Les méthodes, les recommandations, la scientificité de la démarche proposée (établir une revue de la littérature, période de test de la démarche), l'imprègnent. Or l'imbrication de ces figures (figure du patienttrajectoire et figure des démarches qualité) aboutit implicitement sur une nouvelle: même si le terme n'est pas utilisé, le patient est clairement défini comme client. La démarche proposée repose clairement sur des méthodes de démarches qualité orientées client (roue de Deming, analyse de processus, notion d'efficience, de rationalisation). Figure de façade et figure implicite se mêlent donc sous cette notion de patient-client. Dans ces mouvements de convocations de figures, sont ainsi enveloppées des valeurs.

Si dans le texte étudié ci-dessus le pli de la figure du patient-client reste léger, il peut prendre une forme plus prononcée dans des textes qui sortent de la dimension normative pour évoquer la dimension pragmatique d'une telle démarche. Dans un article ${ }^{8}$, une cellule qualité et des membres du personnel soignant font part de leur expérience de la mise en place d'une démarche processus dans leur établissement de santé. Cette approche reprend l'idée du chemin clinique, dans la mesure où elle dresse une cartographie - une 
autre forme de textualisation - du processus centrée sur le patient. Cette représentation spécifique repose sur la mise en schéma de qui fait quoi, pour quoi et pour qui (p. 3). Dans la carte il est intéressant de voir que l'intrant du système est l'usager (qui a des besoins et demande une prise en charge) ; il en est aussi l'extrant (l'usager pris en charge dans les règles de l'art et satisfait). La figure du patient repose sur la formalisation de ses besoins et des moyens pour les satisfaire. Il est appréhendé sur la carte comme une donnée du processus, voire du système. Dans cette focale, le patient est un «livrable» (p.5). La relation patient-organisation se transforme donc en une relation client-fournisseur, selon les principes de la démarche processus. Une nouvelle forme de relation contractuelle s'installe ainsi, qui n'est pas la même que celle relevée pour les associations de patients dans la mesure où dans ce contrat-ci le patient est aussi objet du contrat. Mais l'image de la relation contractuelle est poussée plus loin et aboutit sur un glissement de figure: le patient telle une pièce est livrée de services en services. Chaque service de l'établissement de soin est en charge du livrable-patient, en cela chaque service est fournisseur et client des autres. La figure du client est donc réincarnée, adaptée au personnel qui lui-même devient client... dès lors que devient la figure du patient? Celle sous-entendue d'une pièce, d'un objet qui circule ? Le parcours du patient devient-il une chaîne de montage ?

Comprendre la figure du patient à l'œuvre dans ces textes nécessite ainsi de prendre en compte les valeurs, les principes qu'elle enveloppe et qui l'enveloppent. Une figure est un ensemble de configurations et son autorité dépend des principes qui la constituent. Nous constatons ici un alignement entre la figure-client du texte global de la HAS et celle du texte local de la cellule qualité. La traduction assez fidèle des principes de la HAS laisse à penser que cette figure fait désormais autorité; une autorité qui peut découler de la dimension normative du texte de la HAS.

Il faut néanmoins nuancer la dureté de la figure ainsi présentée : l'article de la cellule présente en effet une vision moins abstraites du patient, ne serait-ce que par la présentation de la spécificité des soins fournis dans cet établissement ou ses nombreuses re-présentations (le patient lui-même, l'accompagnant, l'ambulancier par exemple illustrent ces effets de présence). Il est mis un bémol à l'efficacité de cette démarche processus soulignant sa dimension coûteuse et chronophage. Le redéploiement de l'organisation par rapport à cette figure du patient n'est donc pas sans heurt. Néanmoins, on notera l'alignement avec le texte de recommandations de la HAS sur le chemin clinique avec une traduction assez fidèle de la démarche qualité centrée patient. Notamment, il est accentué la question des écarts où les variances par rapport à la trajectoire scriptée construite par le personnel lui-même sont travaillées. La trajectoire construite, ce schéma type du parcours patient, ordonne (et ordonnance) l'organisation, aligne les membres du personnel les uns aux autres mais néanmoins doit être réajustée s'il y a trop d'écarts. "Se plier à " ne signifie pas implacablement se figer dans une certaine position, se plier c'est aussi savoir se courber et permettre une certaine flexibilité (Deleuze, 1988).

Le chemin clinique présente une figure du patient qui chemine dans l'institution. Or le patient est-il toujours le même tout au long de ce parcours ? Qu'est-ce qui change dans ce pliage et dépliage? Est-ce l'organisation ou le patient? Ou aucun des deux? Dans ces textes qualité est-ce le patient qui est interrogé ou la réorganisation de l'organisation? 


\section{Figures du patient - figures de l'organisation : se " plier à » ou se re-plier?}

Les textes étudiés montrent deux agencements autour de la figure du patient. Si le patient est envisagé dans une figure le plaçant comme le centre d'une réorganisation des performances des établissements de santé dans un esprit d'efficacité, la HAS l'appréhende également dans une relation contractuelle plus symbolique: celle des droits et des devoirs. Ainsi, par exemple, dans le manuel d'accréditation $2010^{9}$ mais aussi dans le communiqué de presse ainsi que dans le guide sur la coopération avec les associations, le patient est présenté comme une figure législative qui a des droits et qui plie l'organisation selon ses devoirs. Si le patient se plie à l'organisation, l'inverse est vrai également.

Dans le manuel d'accréditation, une des thématiques les plus importantes, avec la prise en charge du patient est le «droit» du patient (bientraitance, information, consentement, prise en charge de la douleur, fin de vie). Dans cette figure du droit, de manière implicite, resurgit le patient en tant que personne ${ }^{10}$. S'il y a un contrat entre l'organisation et le patient c'est aussi un contrat moral et éthique. Cette fois c'est le pli du patient qui enveloppe l'organisation dans un ensemble de responsabilités. Si les relations contractuelles client (patient) - fournisseur (organisation) et droits (patients) et devoirs (organisation) peuvent sembler, au premier abord, éloignées, elles sont regroupées dans l'ensemble des textes étudiés. L'enjeu de la figure du patient en tant que personne tient dans cette mise en compatibilité de ces configurations.

\section{Conclusion}

Nous voudrions conclure à deux niveaux : sur les portées pratiques et théoriques de cette étude.

Du point de vue pratique, la multiplicité des figures du patient représente une sorte de défi pour les organisations de soin de santé qui cherchent à les concilier. Dans quelle mesure une organisation peut-elle se maintenir quand elle doit se plier et se déplier selon la variabilité de figures? Tout l'enjeu repose sur l'autorité de ces configurations : entre une autorité légale, une autorité morale, une autorité législative, l'organisation se doit d'appréhender des logiques différentes. Cette variabilité peut être à la fois pénalisante, en tant que contrainte, et salutaire, laissant une marge de manœuvre dans le choix de privilégier l'une ou l'autre. Toutefois, la question qui se pose est de savoir si les établissements de santé ont réellement la possibilité de choisir... De plus que se passe-t-il quand la figure du patient s'incarne? Comment passe-t-on de la textualisation à la pratique? Ne serait-ce pas un nouveau redéploiement?

D'un point de vue théorique, nous avons voulu prolonger les travaux de Cooren (2010) sur la notion de figure et son agentivité dans le discours en abordant un autre support que celui du dialogue: l'écrit. En ce sens, nous suivons l'argument de Fraenkel (2007) concernant la spécificité des textes (ici des figures textualisées) dans la mise en écrit/mise en acte de l'organisation. Toutefois, nous avons voulu montré que figure et écrit ne signifient pas une réalité figée de principes ou de l'organisation. La notion de figure travaillée avec les notions de configuration et de plis nous permet d'avoir une approche 
dynamique du texte. Ainsi, il nous semble que l'agentivité des textes et des figures qu'ils supportent est une clé pour en appréhender une dimension processuelle. Les textes étudiés se présentent comme des agents : ils ordonnancent de nouvelles trajectoires, recourant aux multiples facettes de la figure du patient. Dans ce jeu, aussi bien le patient que l'organisation sont textualisés. Les textes ont ici une forte agentivité en tant qu'inscriptions, prescriptions et sanctions. Ils espacent les différents acteurs des établissements de santé et des hautes instances décisionnelles telles que la HAS. Ainsi le patient et l'organisation se plient aux démarches qualité. Dans notre étude de la figure, c'est moins la figure en elle-même qui nous intéresse que les configurations qui la génère et qu'elle supporte. Les figures sont ainsi porteuses d'une forme d'organisation, un mouvement de pli et de dépli. Cerner les logiques inscrites dans les figures est une clé d'entrée que nous proposons pour déceler une vision processuelle du texte.

31 Saisir les configurations portées par les figures nous permet de comprendre dans quelle mesure le texte véhicule des formes organisationnelles et dans quelle mesure ces figures participent de la construction de l'organisation. Toutefois saisir les figures comme porteuse d'une dynamique induit également de saisir les figures dans leur dynamique : il ne faudrait pas assimiler les figures textualisées comme une forme réifiée de l'organisation. Selon l'approche texte/conversation, ces figures ne sont qu'une stabilisation temporaire remise en acte lors des conversations. Ainsi, les configurations de figures d'un texte sont sujettes à d'autres reconfigurations lors d'autres reprises dans d'autres contextes d'énonciations: les figures textualisées deviennent des figures énoncées. Suivre les formes de mobilisation des figures, déformées et reformées au gré des interactions, serait alors une méthode pour saisir l'effectivité de leur performativité supposée par les textes. Dans le cadre de notre étude, une approche longitudinale nous amènerait, par exemple, à saisir la force normative des figures des textes de la HAS au regard de leur réappropriation par les différents acteurs de l'hôpital, et ainsi de questionner l'autorité des figures. Ces étapes de reconfigurations de figures sont elles aussi constitutives de l'organisation.

\section{BIBLIOGRAPHIE}

Bouzon, A., (2009), Les écrits de travail dans l'activité collaborative de conception. Entre partage des savoirs et bricolage communicationnel, in Semen, $\mathrm{n}^{\circ} 28, \mathrm{pp} .73-100$.

Brassac, C., Fixmer, P., Mondada, L. et Vinck, D., (2008), Interweaving objects, gestures, and talk in context, in Mind, Culture and Activity : An International Journal, vol. 15, n² 2, pp. 208-233.

Brassac, C. et Le Ber, F., (2005), Inscription spatiale d'une activité cognitive collective de représentation de l'espace, in Intellectica, $\mathrm{n}^{\circ}$ 41-42, pp. 181-200.

Castel, P., (2002), Quand les normes de pratiques deviennent une ressource pour les médecins, in Sociologie du travail, $\mathrm{n}^{\circ} 44, \mathrm{pp}$. 337-355. 
Cooren, F., (2006), The organizational world as a plenum of agencies, in F. Cooren, J. R. Taylor et E. J. Van Every (éds.), Communication as organizing : Empirical and theoretical explorations in the dynamic of text and conversation (pp. 81-100), Mahwah, NJ, Lawrence Erlbaum.

Cooren, F., (2004), Textual Agency : How Texts Do Things in Organizationnal Settings, in Organization, $\mathrm{n}^{\circ} 11(3)$, pp. 373-39.

Cooren, F., (2010a), Action and agency in dialogue : Passion, ventriloquism and incarnation, Amsterdam, John Benjamins, 222 p.

Cooren, F., (2010b), Comment les textes écrivent l'organisation. Figures, ventriloquie et incarnation, in Études de communication, $\mathrm{n}^{\circ} 34, \mathrm{pp} .23-40$.

Deleuze, G., (1988), Le pli, Paris, Éditions de Minuit, 191 p.

Fraenkel, B., (2007), Actes écrits et actes oraux : la performativité à l'épreuve de l'écriture, in Études de Communication, $n^{\circ} 1(29)$, pp. 69-93.

Joerges, B. et Czarniawaska, B., (1998), The question of technology, or how organizations inscribe the world, in Organization Studies, $\mathrm{n}^{\circ} 19(3)$, pp. 363-385.

Mayère, A., Grosjean, S. et Bonneville, L., (2010), Rationalisations des organisations hospitalières : des incitations économiques aux injonctions à collaborer, in Sciences de la Société, n 74, pp. 3-13.

Minvielle, E., (2003), De l'usage de concepts gestionnaires dans le champ de la santé. Le cas de la qualité hospitalière, in Revue Française de Gestion, $\mathrm{n}^{\circ}$ 146-5, pp. 167-189.

Morillon, L., (2009), Le journal de communication interne, un agent des enjeux stratégiques organisationnels, in Semen, $\mathrm{n}^{\circ} 28, \mathrm{pp} .33-51$.

Ogien, A., (2009), L'hôpital saisi par la quantification. Une analyse de l'usage gestionnaire de la notion de qualité, in Sciences de la société, $n^{\circ} 76$.

Paccioni, A. et Sicotte, C., (2010), L'accréditation des services en santé de première ligne : l'amorce d'une nouvelle culture organisationnelle ?, in Sciences de la Société, $\mathrm{n}^{\circ}$ 76, pp. 114-133.

Pierru, F., (2009), Les professionnels de santé à l'épreuve de la gouvernance d'entreprise, in Enfances psy, $n^{\circ} 43$, pp. 99-105.

Pontille, D., (2009), Écriture et action juridique. Portrait de l'Huissier de justice en réparateur, in Semen, $n^{\circ} 28$, pp. 15-31.

Rousset, G., (2009), Quelle place pour l'usager dans le système de santé français ?, $77^{\mathrm{e}}$ Congrès International de l'ACFAS, Colloque Organisation et communication au sein de systèmes de santé, 11-12 mai, pp. 34-46.

Sainsaulieu, I., (2007), Normes évaluatives, pluralisme et diversité. Le succès paradoxal des recommandations médicales, in Bouquet B., Jaeger M. et Sainsaulieu I., Les défis de l'évaluation en action sociale et médico-sociale, Paris, Dunod, pp. 109-129.

Vásquez, C., (2010), Espacer l'organisation : trajectoires d'un projet de diffusion de la science et de la technologie au Chili, in Loneux C. et Parent B. (Eds.), Communication des organisations : recherches récentes, Paris, L'Harmattan, pp. 187-196.

\section{NOTES}

1. Dans ce document, le genre masculin est utilisé comme générique, dans le seul but de ne pas alourdir le texte. 
2. Une première ébauche de cet article a été présentée au colloque Mutations des systèmes de santé et communication entre organisations, professionnels, patients et Société civile, ACFAS 2010. Nous tenons d'ailleurs à remercier les organisatrices et participants des échanges et questions qui nous ont permis de poursuivre notre réflexion. Nous remercions également les évaluateurs de cet article ainsi que l'éditeur pour leurs remarques pertinentes qui nous ont permis d'affiner nos arguments.

3. La HAS se définit comme une autorité administrative indépendante à caractère scientifique qui a comme mission d'évaluer les services de soins, de promouvoir les bonnes pratiques, d'améliorer leur qualité, d'informer les professionnels de santé et le grand public, de développer la concertation et la collaboration avec divers acteurs associés au système de santé (http:// www.has-sante.fr/portail/jcms/c_452559/presentation-de-la-has, consulté le 26/02/2010).

4. Il est important de noter ici que la notion de pli telle que nous l'utilisons dans le cadre de cet article est mobilisée en tant qu'image nous permettant de conceptualiser les reconfigurations organisationnelles à partir des mouvements d'inclusion et d'exclusion des figures du patient. Bien que nous nous référions aux réflexions de Deleuze (1988) sur le pli, nous ne cherchons pas à les transposer à l'organisation. Nous nous arrêtons à l'évocation du pli en tant qu'articulateur, objet frontière - l'expression minimale du mouvement que nous explorons à travers les effets de reconfigurations.

5. Haute Autorité de Santé (2008), Cadre de coopération avec les associations de patients et d'usagers, p. 9 (http://www.has-sante.fr/portail/jcms/c_660855/cadre-de-cooperation-avec-lesassociations-de-patients-et-dusagers, consulté le 26/02/2010).

6. http://www.has-sante.fr/portail/jcms/c_611349/la-haute-autorite-de-sante-consulte-lesassociations-de-patients.

7. Haute Autorité de Santé (2004), Chemin clinique Une méthode d'amélioration de la qualité, $44 \mathrm{p}$. (http://www.has-sante.fr/portail/jcms/c_436520/chemin-clinique, consulté le 26/02/2010). Haute Autorité de Santé (2005), Une méthode d'amélioration de la qualité, Chemin clinique Clinical Pathway-Critical Pathway- Inegrated Care Pathway, (http://www.has-sante.fr/portail/jcms/ c_436520/chemin-clinique, consulté le 26/02/2010).

8. Haute Autorité de Santé (2008), Manuel de certification des établissements de santé V2010, $84 \mathrm{p}$. (http://www.has-sante.fr/portail/jcms/c_714928/manuel-v2010-de-certification-desetablissements-de-sante-version-juin-2009, consulté le 26/02/2010).

9. D. Verger, J. Mihura, F. Salle, J. Sarini et V. Colin (2008), Le management par les processus dans un établissement de soins : une démarche utopique?, in Bulletin du cancer, vol. 95, $\mathrm{n}^{\circ} 7$.

10. Ceci participe d'un nouveau modèle du droit français, la démocratie sanitaire. G. Rousset (2009) montre la relativité du modèle au regard de la réalité et le manque d'effectivité des mécanismes actuels de participation des usagers.

\section{RÉSUMÉS}

Participant d'un mouvement de rationalisation des organisations hospitalières, les démarches qualité s'attachent à donner une nouvelle place à cet acteur particulier qu'est le patient. Dans cet article nous cherchons à déceler les différentes figures du patient convoquées (usager, client, participant, expert), comment elles sont configurées par leurs articulations avec l'organisation de soins de santé. Les démarches qualité poussent les organisations de soins de santé à s'ouvrir 
vers cet acteur : à se déplier - se laisser questionner par (et pour) le patient - et à s'y (re)plier - se renfermer, y envelopper le patient. Nous faisons l'hypothèse que l'étude des figures textualisées du patient est une clé pour comprendre les mouvements de pli et de dépli à travers lesquels le patient et l'organisation sont reconfigurés ; les figures du patient poussent ainsi l'organisation à s'« espacer » différemment (Vasquez, 2009). Comment maintenir dès lors l'organisation à travers ces multiples reconfigurations (successives et simultanées)?

As part of the rationalization of healthcare organizations, total quality management seeks to give greater importance to one actor, namely the patient. In this paper, we seek to identify the various representations of the patient (user, customer, member, expert) and how they are configured in relation to the healthcare organization. Quality measures are forcing healthcare organizations to open up to this actor by unfolding themselves on behalf of and for the benefit of the patient and to then fold themselves up again, delimit their boundaries, and wrap up the patient. We propose that the study of the patient's textualized representations is key to understanding the folding and unfolding processes through which the patient and the organization are reconfigured. We argue that the representations of the patient configure the organization in many different ways (Vasquez 2009). The key issue is how to maintain the organization through its multiple successive and concurrent reconfigurations.

\section{INDEX}

Keywords : figures of the patient, quality measure, textual agentivity, organization, text

Mots-clés : figures du patient, démarche qualité, agentivité textuelle, organisation, texte

\section{AUTEURS}

\section{ALEXIA JOLIVET}

C.E.R.T.OP. - Université de Toulouse III

Alexia Jolivet est doctorante au Laboratoire C.E.R.T.O.P. équipe ECORSE, Université Toulouse 3. Ses recherches sont tournées vers la communication en tant que constituante de l'organisation, en travaillant notamment de manière interdépendante les notions de textes (Taylor, 2001) et de travail d'organisation (De Terssac, 2002) ; ses études portent, dans le domaine de la santé, sur les logiques gestionnaires et morales traversant et travaillant la notion de qualité. Adresse électronique : alexia.jolivet@iut-tlse3.fr.

\section{CONSUELO VÁSQUEZ}

Université du Québec à Montréal

Consuelo Vásquez, Ph.D. (Université de Montréal 2009) est professeure au Département de communication sociale Publique à l'Université du Québec à Montréal (UQAM). Ses travaux s'inscrivent dans les approches constitutives de la communication organisationnelle, à partir desquelles elle aborde des questions associées à la mouvance des organisations et aux diverses formes de re-présentations collectives. Elle est membre active du Groupe de recherche Langage, Organisation et Gouvernance de l'Université de Montréal. Elle fait aussi partie de la Chaire en relations publiques et communication marketing et du Centre ComSanté de l'UQAM. Adresse électronique : vasquez.consuelo@uqam.ca. 